\title{
Die Vereinten Nationen und Deutschlands Verantwortung
}

\author{
Alexander S. Neu*
}

Abstract: Germany permanently underscores its bigger responsibility, the importance of the UNO and of international law. Despite this lip service, so far no German government was eager in providing more manpower, material and funds to enable the World Organisation to do its main designated work: to maintain international peace and security. However, Germany is involved in some international military missions - some UN-conducted and some UN-mandated. The most important ones are merely UN-mandated, which means not actually controlled by the UN.

Keywords: Vereinte Nationen, Deutschlands Verantwortung, Auslandseinsätze, UNO-Gewaltmonopol

$\mathrm{D}$ eutschland beteiligt sich derzeit (Stand November 2006) mit ca. 9.150 Soldaten an 11 Auslandseinsätzen:

- vier UN-geführte Einsätze, d.h. unter militärischem Oberkommando der UNO, mit insgesamt etwa 1.100 Soldaten $^{1}$

- fünf UN-mandatierte Einsätze, d.h. unter militärischem Oberkommando eines Staates oder einer regionalen internationalen Organisation mit UNO-Mandat, mit etwa 7.300 Soldaten ${ }^{2}$ sowie

- zwei völkerrechtlich fragwürdige US-geführte AntiterrorKriegseinsätze: »Operation Enduring Freedom « und »Operation Active Endeavour « mit etwa 550 Soldaten $^{3}$

Hinzu kommt, dass weitere Einsätze bzw. die Ausdehnung bestehender vor dem Hintergrund der außer Kontrolle geratenden Konflikte auf dem afrikanischen Kontinent nicht auszuschließen sind. ${ }^{4}$

Es wird deutlich, wie heterogen und unübersichtlich die Einsatz- und Legitimationsformen sind. Damit wird die effektive parlamentarische Kontrolle deutscher Außen- und Sicherheitspolitik erschwert.

Zählt man zu den Befürwortern des Primats einer stabilen und übersichtlichen internationalen Rechts- und Friedensordnung, dann muss es darum gehen, der UNO formal und faktisch die singuläre Verantwortung für das globale System der kollektiven Sicherheit zu erhalten. Dazu müssen eine Standardisierung der militärischen Einsatz- und Legitimationsformen sowie umfassende polizeiliche Kapazitäten für die UNO gefordert werden, um ihr ein berechenbares und effektives Handeln zu ermöglichen.

Angesichts der steigenden Zahl der Beteiligungen Deutschlands an militärischen Einsätzen ist es vordringlich, Antworten auf folgende Fragen zu finden:

\footnotetext{
* Dr. phil. Alexander S. Neu, Referent für Sicherheitspolitik der Bundestagsfraktion DIE LINKE und Journalist, Berlin. Der Beitrag gibt ausschließlich die persönliche Meinung des Autors wieder.

1 UN-geführte Einsätze: UNMIS, UNOMIG, UNMEE und UNIFIL.

2 UN-mandatierte Einsätze: ISAF, KFOR, EUFOR (ALTHEA), AMIS, EUFOR RD Congo.

3 »Operation Enduring Freedom« reicht von Afghanistan bis ans Horn von Afrika. »Operation Active Endeavour « findet im Mittelmeer statt.

4 Spiegel Online, 2. Juli 2006: »Jung erwägt weitere Afrika-Einsätze« http:// www.spiegel.de/politik/deutschland/0,1518,424644,00.html / Franz Josef Jung, Klares deutsches Interesse, Interview mit dem Bundesverteidigungsminister, in: »Der Spiegel«, 24.07.2006.
}

1. Sind zivile und polizeiliche Krisenpräventions- und Konfliktregulierungsinstrumente nicht doch effektiver als das militärische Instrument?

2. Falls diese nichtmilitärischen Instrumente nicht greifen, welche politischen und strukturellen Voraussetzungen müssten generell vorliegen, um Auslandseinsätze der Bundeswehr mit der Maßgabe der Stärkung der UNO sowie der internationalen Rechts- und Friedensordnung zu befürworten?

Die erste Frage könnte mittels einer umfassenden empirischen Analyse der abgeschlossenen und laufenden militärischen Operationen zumindest partiell beantwortet werden. Eine solche Analyse ist jedoch nicht Gegenstand dieses Beitrags. Er diskutiert ausschließlich die zweite Frage. Hierbei wird zunächst die offene und schleichende Delegitimierung der UNO, die alleine die Verantwortung für das globale System kollektiver Sicherheit trägt, durch Deutschland und die USA beschrieben. Der zweite Abschnitt erläutert die zentralen Defizite der UNO, die diese Delegitimierungsbemühungen begünstigen. Der dritte Abschnitt skizziert das widersprüchliche Verhältnis von Pazifismus und UNO-Gewaltmonopol. Abschließend werden politische und militärische Beitragsformen sowie politische Bedingungen formuliert, mit denen Deutschland die UNO substanziell stärken könnte.

\section{Die Relativierung der UNO als singulärer Träger des Gewaltmonopols}

Alle von Deutschland mitgetragenen militärischen Maßnahmen werden mit einer gewachsenen Verantwortung in der Welt, mit erforderlicher Bündnissolidarität und als im Rahmen des geltenden Völkerrechts bzw. notwendig zur Stärkung der UNO erklärt.

Aber nicht nur das Verständnis des militärischen Instrumentariums ist in der politischen Klasse Deutschlands einem fundamentalen Wandel unterworfen, sondern auch zunehmend die Frage der Legitimationsquelle seiner Anwendung.

Ein interessanter Indikator für den Wandel des Verständnisses der Legitimationsquelle zur Anwendung militärischer Zwangsmaßnahmen und somit der sich herausbildenden mangelnden Vertragstreue gegenüber der UNO-Charta ist - neben der politischen Praxis und der höchstrichterlichen Rechtsprechung des Bundesverfassungsgerichts zum Charakter der NATO - wie bereits oben ausgeführt, der schwarz-rote Koalitionsvertrag: »Wir wollen einen effektiven Multilateralismus, bei 
dem die internationalen Organisationen zum Zuge kommen, die für die Lösung eines konkreten Problems am besten geeignet sind. Den Vereinten Nationen kommt weiterhin eine Schlüsselrolle für die Wahrung des Friedens (...) zu«. ${ }^{5}$ Es sind rhetorisch verklausulierte und weiche Aussagen wie diese, die eine Interpretation der Akzeptanz der singulären und obersten Verantwortung der Weltorganisation für den Weltfrieden und die internationale Sicherheit - einschließlich des UN-Gewaltmonopols - erlauben. Ein explizites Bekenntnis zum UNGewaltmonopol hingegen sucht man vergeblich. Stattdessen der Verweis auf internationale Organisationen, der zumindest die Intention zur Diversifikation der Legitimationsquellen zu militärischen Zwangsmaßnahmen nicht ausschließt.

Die oben zitierten Textpassagen des Koalitionsvertrags könnten als ein Hinweis gewertet werden, Deutschland einem in den USA diskutierten und von ihr bereits praktizierten außenpolitischen Kurs anzunähern ${ }^{6}$ : Offener Multilateralismus à la carte oder, wie es der bekannte US-amerikanische Professor für internationale politische Ökonomie Francis Fukuyama ausdrückt: einen »Multi-Multilateralismus « ${ }^{7}$. Hierbei handelt es sich um einen »freien Markt der internationalen Zusammenarbeit ", in dem eine Vielzahl "geografisch und funktional sich überschneidender« internationaler Institutionen existiert, die dem Westen letztlich als toolbox für ein »zweckmäßiges Handeln« dienen. Die UNO muss, so der Tenor, zu einer internationalen Institution unter vielen reduziert werden. Begründet wird diese rein machtpolitische, die UNO in die politische Bedeutungslosigkeit verbannende Forderung damit, sie sei »weder effizient noch legitimiert «, wenn es um Fragen der internationalen Sicherheit gehe. Dabei verschweigt Fukuyama den wesentlichen Grund für die bisweilen mangelhafte Effizienz der Weltorganisation: dass eine Organisation immer nur so effizient sein kann, wie die sie tragenden Mitglieder ihr auch die notwendigen Kapazitäten zur Erfüllung der ihr zugeschriebenen Aufgaben zur Verfügung stellen.

Nicht weniger bedenklich ist die Behauptung der mangelnden Legitimität der UNO. Die Legitimität internationaler Institutionen leitet Fukuyama nicht aus der Souveränität ihrer Mitgliedstaaten, sondern aus deren demokratischer Verfasstheit ab. In einer Glorifizierung des eigenen westlichen Demokratieverständnisses unterstellt er der NATO, im Gegensatz zur UNO, die Legitimität zu militärischen Maßnahmen wie beispielsweise den Angriffskrieg gegen Jugoslawien im Jahr 1999: »Die NATO hat weniger Legitimitätsprobleme als die Vereinten Nationen. Alle ihre Mitgliedstaaten sind echte liberale Demokratien (...). « 8 Mit einem derart wertbeladenen außenpolitischen Verständnis steht Fukuyama nicht alleine da. ${ }^{9}$ Es zeichnet sich in der westlichen, vor allem angelsächsischen Welt vielmehr ein gefährlicher Trend ab, nur noch Staaten mit westlichen Demokratiemodellen als gleichberechtigte Akteure zu akzeptieren. ${ }^{10}$

5 Koalitionsvertrag zwischen CDU, CSU und SPD für die 16. Wahlperiode des Deutschen Bundestages, Gemeinsam für Deutschland. Mit Mut und Menschlichkeit., 18. November 2005, S. 66f.

6 Insbesondere verweist die »National Security Strategy (NSS) der US-Regierung aus dem Jahre 2002 sowie die Neuauflage von 2006 auf die geringe Relevanz der UNO für die US-Außenpolitik.

7 Francis Fukuyama, So regieren wir die Welt, in: »Cicero - Magazin für politische Kultur «, 4/2006, S. 42-47.

8 Francis Fukuyama, a.a.O.

9 Vgl. auch Kagan, Robert: Macht und Ohnmacht. Amerika und Europa in der Neuen Weltordnung. Berlin 2003.

10 Nassauer, Otfried, »Vereinte Nationen und NATO - Ein Deja Vu im geographischen Neuland«, BITS Research Note 99.2, Berlin, 1999.

\section{Zentrale Defizite der UNO}

Was aber ist die Achillesferse der UNO mit der versucht wird, eine demokratietheoretisch und effizienzbasierte Delegitimierung abzuleiten? Im Wesentlichen handelt es sich um zwei grundlegende und miteinander verbundene Schwächen:

1. Die bis heute von den Mitgliedstaaten immer noch nicht umgesetzte Verpflichtung, der UNO entsprechende militärische Kapazitäten an die Hand zu geben, damit diese auch das Gewaltmonopol effektiv materialisieren kann. Es dürfte seitens der Gründungsväter und -mütter der UNO kein Zufall gewesen sein, dass sie das formale Gewaltmonopol (Kapitel VII, Art. 39-42) in ein und dasselbe Kapitel der Charta geschrieben haben, wie das materielle Gewaltmonopol (Kapitel VII, Art. 43-47).

2. Das demokratische Defizit der UNO aufgrund mangelnder demokratischer Strukturen, die eine unzureichende Legitimität ihrer Beschlüsse und des materiellen Gewaltmonopols behaupten lässt und eine effektivere Kontrolle der UNOGremien erschwert.

Eine Gelegenheit, beide Defizite aufzuarbeiten, hätte der UNGipfel zum 60. Jahrestag der Gründung der UNO im Jahr 2005 geboten. Im Vorfeld dieses Gipfels wurde die so genannte Hochrangige Beratungsgruppe für Bedrohungen, Herausforderungen und Wandel (High Level Panel on Threats, Challenges and Change) einberufen, um Reformvorschläge zu entwickeln. Das Ergebnis dieser Kommission war interessant und wurde im Wesentlichen durch UNO-Generalsekretär Kofi Annan in seinen Empfehlungen unter dem Titel »In größerer Freiheit: Auf dem Weg zu Entwicklung, Sicherheit und Menschenrechten für alle« für den anstehenden Reformgipfel übernommen. ${ }^{11}$ Allerdings blieben die eigentlich essentiellen Fragen nach der Demokratisierung der UNO-Strukturen sowie die Stärkung des materiellen UNO-Gewaltmonopols nicht nur außen vor, UNO-Generalsekretär Annan forderte sogar in gänzlich unverständlicher Weise - im Zuge der Bereinigung der UNO-Charta von nicht mehr zeitgemäßen Rechtsnormen - die Streichung selbst jener Artikel, die das materielle Gewaltmonopol erst ermöglichen. ${ }^{12}$ Eine auf autonomen militärischen oder auch nur polizeilichen Kapazitäten basierende und somit politisch handlungsfähige UNO scheint damit für Annan nicht mehr zeitgemäß zu sein. Damit hat sich Annan offen gegen die Position seines Amtsvorgängers Boutros Boutros Ghali gestellt, der 1992 in seiner »Agenda für den Frieden « eine ständige, den Vereinten Nationen direkt zu unterstellende Eingreiftruppe gemäß Kapitel VII forderte. ${ }^{13}$

Stattdessen setzt Annan auf outsourcing, d.h. darauf, militärische Maßnahmen lediglich zu mandatieren und an Dritte zu delegieren.

11 Hochrangige Beratungsgruppe für Bedrohungen, Herausforderungen und Wandel: Eine sicherere Welt: Unsere gemeinsame Verantwortung, Vereinte Nationen, Generalversammlung, Dokument A/59/565, 2004, unter: http:// www.dgvn.de/pdf/High_Panel_Report_deutsch.pdf. In größerer Freiheit: Auf dem Weg zu Entwicklung, Sicherheit und Menschenrechten für alle, UNDocument: A/59/2005, 21.03.2005, deutsche Fassung unter: http://www. un.org/depts/german/gs_sonst/a-59-2005-exesumm.pdf.

12 In größerer Freiheit, a.a.O., Punkt 219, S. 52.

13 Agenda für den Frieden, Vorbeugende Diplomatie, Friedensschaffung und Friedenssicherung, A/47/277 - S/24/111, 17.06.1992, deutsche Fassung unter: http://www.un.org/Depts/german/friesi/afried/afried-1.htm. 


\subsection{UN-mandatierte vs. UN-geführte Operationen}

In der Tat kann der UNO-Sicherheitsrat auf der Grundlage des Artikels 53 der UNO-Charta einzelne Staaten oder regionale Einrichtungen mit deren Einverständnis »unter seiner Autorität in Anspruch« nehmen, um »Zwangsmaßnahmen« durchzusetzen. Ungeklärt bleibt hierbei indes die präzise Definition dieser »Autorität«. Sollen die ad hoc bereitgestellten Truppen für die militärischen Zwangsmaßnahmen unter internationalem Oberkommando (UN-geführt) stehen, womit die Autorität der UNO aufgrund der institutionellen Einheit von politischer Autorität und militärischer Befehlskette mehr oder minder gut abgesichert wäre? Oder sollen die Truppen unter nationalem Oberkommando (UN-mandatiert), d.h. mit eigenständiger militärischer Befehlskette jenseits der UN-Strukturen operieren, wodurch einer interessendeterminierten Mandatsauslegung und -implementierung durch die Truppen stellenden Staaten Tür und Tor geöffnet würde und die UN-Autorität zur Farce werden könnte?

Woher Annan den Optimismus einer aufopfernden Selbstlosigkeit der die Truppen stellenden Staaten, zum einen mit Blick auf die Zielregion der möglichen Intervention, und zum anderen mit Blick auf die Ausdehnung ihres außenpolitischen Handlungsspieltraums jenseits der UNO angesichts der bislang gemachten Erfahrungen nimmt, bleibt sein Geheimnis.

Ein Blick in die jüngere Vergangenheit seit Ende des Ost-WestKonflikts gibt jedenfalls wenig Anlass zu Optimismus:

- So wurde die Befreiung Kuwaits durch eine multinationale Truppe unter US-Oberkommando durchgeführt. Inwieweit die Luftangriffe der UN-mandatierten multinationalen Truppe und erst recht die Bodenoffensive auf irakischem Territorium mit erheblichen menschlichen und materiellen Schäden (so genannten Kollateralschäden) erforderlich war, um die Souveränität Kuwaits (UN-Resolution 660) auch unter Zuhilfenahme militärischer Zwangsmittel wiederherzustellen (UN-Resolution 678), ist mehr als fraglich und bedürfte einer rechtlichen Klärung. Eine noch offensichtlichere und vollständige Verselbstständigung der Alliierten unter USFührung stellte die Errichtung der so genannten Flugverbotszonen im Süden und Norden des Irak dar. Diese Maßnahme war eine Selbstmandatierung, da sie sich nicht aus der UN-Resolution 688 herleiten ließ. Zwar wurde in besagter Resolution die gewaltsame Unterdrückung von Teilen der irakischen Bevölkerung verurteilt und der Irak zur sofortigen Einstellung dieser Repressionsmaßnahmen aufgefordert, militärische Zwangsmaßnahmen wurden damit jedoch keineswegs autorisiert. Die von den USA und ihren Verbündeten deklarierten Flugverbotszonen verletzten somit über viele Jahre die Souveränität des Irak. Büßte also die UNO mit der Mandatierung (UN-Resolution 678) der Alliierten zur Befreiung Kuwaits zunächst »nur « die vollständige Kontrolle über die militärische Operation ein und wurde de facto zum Mandatsbeschaffer degradiert, so mandatierten sich die USA mit der unilateralen und somit völkerrechtswidrigen Errichtung der Flugverbotszonen bereits selbst und marginalisierten die UNO als den eigentlich verantwortlichen Akteur.

- In Bosnien-Herzegowina wurde wegen des Scheiterns der UN-geführten Mission UNPROFOR letztlich 1995 eine US/
NATO-geführte und UN-mandatierte Mission (IFOR/SFOR) etabliert, die auf wundersame Weise über alle die erforderlichen Kompetenzen (»robustes Mandat«) und Instrumente verfügte, die der UN-geführten Mission UNPROFOR von denselben Staaten zuvor nicht eingeräumt worden waren. Die auf diese Weise geschaffene multilaterale Alternative zu UN-geführten Operationen führte im Ergebnis zu einer signifikanten Schwächung der UNO-»Autorität«.

- Schon fast zwangsläufig musste der Schwächung der »Autorität « die faktische Aufhebung ihrer »Hauptverantwortung für den Weltfrieden« folgen: die völkerrechtswidrigen Angriffskriege der US-geführten NATO gegen Jugoslawien 1999 und die von den USA geführte »Koalition der Willigen« gegen den Irak 2003. Beide militärischen Maßnahmen haben nicht nur wie im Falle Bosnien-Herzegowinas eine alternative militärische Operationsform zu UN-geführten Missionen geschaffen, sondern gleich die gesamte UNO als Trägerin des Gewaltmonopols für die Fälle entsorgt, in denen der UNO-Sicherheitsrat nicht »willig « war, ein Mandat auf der Grundlage von Artikel 53 der UNO-Charta für eine "Zwangsmaßnahme « abzusegnen - und dies mit Beteiligung Deutschlands. Dass es sich hierbei nicht nur um zwei »Ausrutscher « handelte, sondern dieses Vorgehen zum Programm erhoben wird, wurde sogar mit dem »Neuen Strategischen Konzept « der NATO aus dem Jahre 1999 dokumentiert. ${ }^{14}$

- Der Entwurf des Weißbuchs 2006 der Bundesregierung degradiert die UNO ebenfalls zu einem Mandatsbeschaffer: »Die einzigartige Bedeutung der Vereinten Nationen besteht darin, einen notwendig werdenden Einsatz militärischer Gewalt mit der völkerrechtlichen Legitimität zu versehen. «15 Diese Aussage ist in dem offiziell verabschiedeten Weißbuch zwar gestrichen, liefert jedoch einen interessanten Einblick in die Denkweise. Allerdings ist mit der Streichung jener Aussage keine Entschärfung vorgenommen worden. Im Gegenteil: Denn gestrichen wurde auch die in dem Entwurf explizit verankerte Bindungsklausel an die Beschlüsse des UNO-Sicherheitsrats, wodurch wohl dem bereits erwähnten »Neuen Strategischen Konzept« der NATO Rechnung getragen werden soll: wenn möglich mit, wenn nötig ohne UNOLegitimation. ${ }^{16}$

Letztlich bleibt festzustellen, dass Artikel 53 der UNO-Charta, die dem UNO-Sicherheitsrat die militärische Handlungsfähigkeit quasi indirekt garantieren soll, sich realiter als Axt gegen die Fundamente der UNO als System gegenseitiger kollektiver Sicherheit selbst erweist.

\subsection{Demokratisierung der UNO}

Nicht weniger machtpolitisch entlarvend ist das Verhalten des Westens in Fragen der Demokratisierung der UNO-Strukturen. Reformüberlegungen, die sowohl die Abstimmungsmechanismen und die Staatenrepräsentanz im UNO-Sicherheitsrat als auch

14 Das Strategische Konzept des Bündnisses, www.nato.int/germany/docu/ p99-065d.htm.

15 Weißbuch - Entwurf - http://www.geopowers.com/Machte/Deutschland/ doc_ger/vorl._WB_2006.pdf, S. 35 .

16 Weißbuch 2006 - zur Sicherheitspolitik Deutschlands und zur Zukunft der Bundeswehr, Berlin, S. 57. 
die Einflussmöglichkeiten der UNO-Generalversammlung auf den UNO-Sicherheitsrat zum Gegenstand haben, scheitern am Unwillen der im UNO-Sicherheitsrat vertretenen Großmächte, ihre Entscheidungsprivilegien und Handlungsspielräume zu Gunsten umfassend demokratischer Partizipation aufzugeben. Spätestens in Fragen der Demokratisierung der internationalen Beziehungen, auch unter Einbeziehung zivilgesellschaftlicher Gruppen, finden sich die demokratischen Staaten plötzlich in erstaunlicher Einträchtigkeit mit der von ihnen als zweifelhaft bezeichneten Demokratie Russland und dem undemokratischen chinesischen Regime. Als jüngstes Beispiel ist hier das Verhalten der USA im Vorfeld und während des UN-Gipfels im September $2005 \mathrm{zu}$ nennen: So präsentierten die USA einen mehrere 100 Punkte umfassenden Änderungskatalog zum mühevoll erarbeiteten Reformpaket, das den Staaten auf dem UNO-Gipfel im September 2005 vorgelegt werden sollte. Mit Blick auf die Generalversammlung, dem repräsentativsten, da nahezu alle Staaten der Erde umfassenden, legitimsten und demokratischsten Organ der UNO, versuchten die USA »die Bezüge auf die Zentralität der Versammlung, ihre Funktion bei der Kodifizierung internationalen Rechts und schlussendlich ihre Autorität zu streichen ${ }{ }^{17}$

Dabei ist das Thema der Demokratisierung internationaler Institutionen umso wichtiger, je mehr die UNO auf Betreiben einiger westlicher Staaten ihr klassisches Handlungsfeld der kollektiven Sicherung des Friedens auf zwischenstaatlicher Ebene um die innerstaatliche Ebene erweitert und somit die Souveränität der Staaten partiell durchlöchert. ${ }^{18}$

Der Grundpfeiler des internationalen Rechts, das absolute Souveränitätsprinzip, soll durch ein neues, konditioniertes Souveränitätsverständnis relativiert werden, um »humanitäre Interventionen « zu ermöglichen. Die intellektuelle Vorarbeit leistete die so genannte International Commission on Intervention and State Sovereignty (ICISS) ${ }^{19}$. Die Anregungen wurden später durch das von UNO-Generalsekretär Kofi Annan eingesetzte High Level Panel und in seinen eigenen Empfehlungen übernommen und weitergedacht. Demnach besäße kein Staat das ausschließliche Abwehrrecht (Verbot der Einmischung in die inneren Angelegenheiten) gegenüber anderen Staaten, sondern nur eine konditionale Souveränität, wonach der Staat eine besondere Fürsorgepflicht gegenüber seinen Bürgern haben müsse. Könne oder und wolle er diese nicht gewähren, so habe die internationale Staatengemeinschaft das Recht zu intervenieren. Hiermit soll die »humanitäre Intervention« zu einem eigenen Rechtsinstitut aufgewertet werden. Zwar solle die Quelle der Legitimation zur Gewaltanwendung auch hier beim UNO-Sicherheitsrat liegen, alternativ aber auch bei der UNO-Generalversammlung, sollte der Sicherheitsrat sich nicht in entsprechendem Maße mit der Notwendigkeit einer »humanitären Intervention « beschäftigen, so zumindest der ICISS-Report und abgeschwächt auch der Bericht des High Level Panel. ${ }^{20}$ In den Empfehlungen Annans ist die UNO-Ge-

17 Bennis, Phyllis, »Eine Kriegserklärung: John Bolton und die UNO«, in: Wissenschaft und Frieden, »60 Jahre Vereinte Nationen«, 4/2005.

18 Weißbuch 2006, S. 57.

19 ICISS: The Responsibility to Protect: Report of the International Commission on Intervention and State Sovereignty, Ottawa: International Development Research Centre, Dezember 2001.

20 ICISS, a.a.O., Punkt 6.7, S. 48. / Report of the High Level Panel on Threats, Challenges and Change, a.a.O., Punkt 208, S. 67. neralversammlung als mögliche Legitimationsquelle für »humanitäre Interventionen « bereits gänzlich verschwunden. Während allenthalben über die Notwendigkeit der Berechtigung zur »humanitären Intervention « und sogar über darüber hinausgehende Interventionsberechtigungen (»demokratische Intervention « etc.) diskutiert wird, wird es auffällig ruhig, wenn es um die Frage einer möglichst breiten demokratischen Legitimation und Kontrolle - zum Beispiel in Form der UNOGeneralversammlung - für die die Souveränität der Staaten und somit das zentrale Prinzip des internationalen Rechts in Frage stellenden Zwangsmaßnahmen geht. ${ }^{21}$

Eine solch schwerwiegende und die internationale Stabilität durchaus gefährdende Frage, ob das Gewaltverbot der UNOCharta um weitere Ausnahmen in Form der »humanitären « oder gar der »demokratischen Intervention« als Rechtsinstitut erweitert werden soll, darf nicht durch bloße Behauptung eines völkergewohnheitsrechtlichen Prozesses westlicher Demokratien dem Rest der Welt aufgedrückt werden. Die internationale Staatengemeinschaft muss sich um einen Konsens bemühen. Darüber hinaus sollte es hierzu keine nur prinzipielle Entscheidung zur Etablierung des Rechtsinstituts der »humanitären « oder anderweitig begründeten Intervention mit dann ausschließlicher Entscheidungskompetenz des UNO-Sicherheitsrats geben, da dies die Missbrauchsgefahr zumindest nicht verringert.

Wenig hilfreich hinsichtlich der notwendigen Transparenz, Kontrolle und Legitimation für derartige Interventionen ist hierzu die Abschlusserklärung (Resolution A/RES/60/1) der UNO-Generalversammlung aus dem Jahre 2005 zum 60. Jahrestag der Gründung der Vereinten Nationen. Darin wird bereits die »humanitäre Intervention « unter der Überschrift $»$ Responsibility to protect from genocide $\left(. . . \ll^{22}\right.$ als Prinzip akzeptiert und der konkrete Einzelfallbeschluss dem UNO-Sicherheitsrat überantwortet.

Mit dieser Akzeptanz ist freilich der zweite Schritt, der die Souveränität der Staaten relativierenden erweiterten Befugnisse des UNO-Sicherheitsrats, vor dem ersten, der erforderlichen Demokratisierung und der damit einhergehenden größeren Legitimität der UNO zur Intervention in die inneren Angelegenheiten, gemacht worden.

Auf diese Weise hat die UNO-Generalversammlung ein wichtiges Druckmittel gegenüber dem UNO-Sicherheitsrat zum Erreichen ernsthafter demokratischer Reformen der Weltorganisation aus der Hand gegeben. Angesichts der Reformunwilligkeit der im UNO-Sicherheitsrat vertretenen Großmächte bleibt die UNO-Generalversammlung somit auch weiterhin bei wichtigen politischen Entscheidungen Zaungast.

Die größtmögliche demokratische Legitimation für jeden Einzelfall ist jedoch vonnöten, um einerseits den interessenmotivierten Missbrauch durch die Großmächte zu minimieren und andererseits die größtmögliche Akzeptanz für einen solchen Einsatz herbeizuführen.

21 Peter Hilpod und Gudrun Zagel, Das Gewaltverbot auf dem Prüfstand: Von der NATO-Intervention im Kosovo zu den aktuellen UN-Reformvorschlägen, in: Sicherheit und Frieden (S+F), 1/2006, S. 38-44.

22 Sixtieth Session of the UN-General Assembly, Resolution: A/RES/60/1, Punkt 139, 2005. 


\section{Deutschlands Rolle zur Stärkung der UNO}

Die vorangegangenen Ausführungen haben verdeutlicht, dass ein materiell impotentes UN-Gewaltmonopol, den Großmächten zum Vorteil gereicht: Entweder versuchen sie, die UNO auf einen Mandatslieferanten zu reduzieren, oder sie handeln direkt selbsternannt im Namen der internationalen Gemeinschaft an derselben vorbei. In beiden Fällen handelt es sich um eine eigennützige Substituierung originärer UNO-Funktionen.

Die UNO muss daher polizeiliche und militärische Instrumente an die Hand bekommen, mit denen sie demokratisch kontrolliert globale Sicherheit durchsetzen kann. Wer eine starke UNO will, kann schwerlich pazifistisch-dogmatisch argumentieren. Wer dennoch an dieser widersprüchlichen Position festhält, befindet sich unfreiwillig im selben Boot mit denjenigen, die eine schwache UNO zu Gunsten einer naturrechtlichen Machtordnung befürworten. Wer das internationale Recht und die UNO stärken will, muss jenseits von Lippenbekenntnissen umfassende polizeiliche Kapazitäten bereitstellen und auf die Umsetzung der Artikel 43-47 der UNO-Charta drängen, die die Schaffung ständiger UN-Militärkapazitäten behandeln.

\subsection{Deutschlands militärischer Beitrag}

Deutschland kann als Beispiel vorangehen und der UNO zu Lasten nationaler, europäischer und transatlantischer Militärpotenziale personelle und materielle militärische Kapazitäten zur Verfügung stellen.

Einen ersten, jedoch bei weitem nicht ausreichenden Schritt hat Deutschland mit der Unterzeichnung des Memorandum of Understanding über das Standby Arrangements System im Jahr 2000 gemacht. ${ }^{23}$ Dieses Abkommen sieht vor, dass Deutschland gewisse militärische Fähigkeiten für die UNO vorhält. Auf Anfrage der UNO kann Deutschland unter Berücksichtigung des souveränen Entscheidungsvorbehalts über die Entsendung militärischer Kapazitäten entscheiden. Allerdings zeigt die Quantität der von Deutschland zur Verfügung gestellten Kapazitäten, dass es sich mehr um eine Alibiveranstaltung, denn um eine wirkliche Stärkung der UNO handelt. In erster Linie werden Kommunikations- und Logistikfähigkeiten vorgehalten, deren Aktivierungszeitraum um die 30 Tage betragen soll. Zwar werden Protection Force Elements aufgelistet. Diese sollen primär für friedenserhaltende Operationen mit unter Umständen einem robusterem Mandat auch unter Kapitel VII eingesetzt werden können. Die quantitative Unterordnung dieser der UNO zur Verfügung zu stellenden militärischen Kapazitäten gegenüber den als prioritär erachteten Verpflichtungen mit Blick auf die NATO und die EU wirft ein Licht auf die mangelnde Ernsthaftigkeit der damals rot-grünen Bundesregierung, die UNO wirklich zu stärken.

Es würde für Deutschland kein Problem darstellen, feste und umfangreiche UN-Kontingente vorzuhalten, die bei Bedarf binnen einer Woche der UNO für UN-geführte Operationen zur Verfügung gestellt werden könnten.

23 Memorandum of Understanding between the Government of the Federal Republic of Germany and The United Nations concerning Contributions to the United Nations Standby Arrangements System, New York, 1. November 2000.
Ebenso wenig wäre es für Deutschland ein Problem, der UNO ständige Aufklärungs- und Kommunikationsstrukturen (z.B.: Satelliten und andere Informationstechnologien) zu überantworten. Die UNO wäre dann nicht mehr auf die Informationsund Nachrichtenzuflüsse einzelner Staaten angewiesen, um sich ein Bild von Krisenregionen zu machen. Sie könnte auf eigene (unmanipulierte) Informationen zurückgreifen, um ein objektives Lagebild als Grundlage politischer Entscheidungen und möglicher operativer Planungen zu erhalten.

\subsection{Deutschlands politischer Beitrag - UN-ge- führte versus UN-mandatierte Operationen}

Eine Beteiligung Deutschlands an konkreten Maßnahmen gegen staatliche oder nichtstaatliche Akteure, die die Wahrung des Weltfriedens und der internationalen Sicherheit gefährden, sowie bei innerstaatlichen Konflikten sollte nur unter UNO-Führung stattfinden. Die politische und operative Verantwortung für jeden militärischen Einsatz muss permanent in den Händen des UNO-Sicherheitsrats und des UNO-Generalstabsauschusses liegen.

Das häufig zu hörende Argument, eine UN-geführte Operation sei weniger effektiv und man müsse daher auf potente Staaten oder Bündnisse zurückgreifen, um für Sicherheit und Frieden zu sorgen, verfängt nicht. Denn erstens könnten - und müssten laut UNO-Charta (Kapitel VII; Art. 43 und 45) - die Großmächte die UNO mit entsprechenden militärischen Kapazitäten ausstatten und zweitens müsste das UN-Mandat für UN-geführte Operationen entsprechend formuliert werden. Dass dies nicht so ist, zeigt beispielsweise die UN-geführte Mission MONUC in der DR Kongo. Anstatt die Mission um erforderliche Kapazitäten auszubauen, wird kurzerhand eine Parallelmission, die UN-mandatierte EU-Mission »EUFOR RD Congo «, entsandt, womit indirekt die Unfähigkeit von MONUC unter Beweis gestellt werden soll.

Auch der Fall der bosnischen Stadt Srebrenica und das Massaker an mehren tausend Bosniaken wird gerne als Beleg für die Unfähigkeit der UNO und die Notwendigkeit einer starken NATO oder EU-Truppe herangezogen. Das in Srebrenica eingesetzte niederländische UNO-Kontingent musste tatenlos mit ansehen, wie die bosnischen Männer von den bosnischen Serben weggeschafft wurden. Als offizielle Begründung für das vermeintliche Versagen der UNO wurde die unzureichende Informationslage über einen bevorstehenden Angriff der bosnischen Serben genannt. Hingegen existieren umfangreiche und aufwändige Rechercheergebnisse, die besagen, dass bereits mehrere Monate vor dem Angriff der UNO entsprechende Informationen vorgelegen hätten. Auf Grundlage dieser Erkenntnisse hätte es konkrete Pläne des Department of Peacekeeping Operations (DPKO) der UNO gegeben, die in der Nähe von Srebrenica stationierten dänischen UNO-Truppen nach Srebrenica zu verlegen, um die Enklave zu schützen. Allerdings sei die Umsetzung dieses Vorhabens an den Mitgliedern des UNO-Sicherheitsrats, allen voran den USA, die den Plan abgelehnt hätten, gescheitert. ${ }^{24}$ Versagt

24 Huub Jaspers, Recherchieren am Extrem - das Massaker von Srebrenica, Vortrag bei der Winterakademie des Deutschen Journalistenverbandes, Sarajevo, 03.2005, in: http://www.bits.de. 
hat auch hier nicht die UNO als Institution, denn die Verwaltungsebene, hier das DPKO, hat frühzeitig reagiert. Vielmehr haben die Partikularinteressen der im UNO-Sicherheitsrat vertretenen Großmächte die UNO daran gehindert, ihren Auftrag zu erfüllen. Dass die Großmächte ihre Interessenpolitik selbst bei UN-geführten Einsätzen mit so desaströsen Konsequenzen verfolgen können, unterstreicht einmal mehr die Notwendigkeit einer Demokratisierung der UNO-Strukturen.

Deutschland muss angesichts dessen auf die Demokratisierung der UNO drängen und dazu konkrete Vorschläge unterbreiten. Wenn überhaupt, kann nur eine demokratisch legitimierte UNO durch Kontrolle ihrer Organe die virulente Missbrauchsgefahr reduzieren und auch die Legitimität für sich beanspruchen, die inneren Angelegenheiten von Staaten, die gröbste Menschenrechtsverletzungen begehen, zu internationalen Angelegenheiten zu erklären.

\subsection{Maßnahmen zu Demokratisierung der UNO}

Es ist jedoch davon auszugehen, dass - angesichts der derzeitigen internationalen Machtkonstellation - eine Demokratisierungsreform der UNO noch lange auf sich warten lassen wird. Hinzu kommt, dass diese Vorgabe im Gegensatz zu der Forderung der überfälligen Umsetzungsverpflichtung militärischer UNO-Kapazitäten (Art. 43 UN-Charta) noch nicht einmal ein Rechtselement der UNO-Charta darstellt.

Bis zum Zeitpunkt einer tatsächlichen und umfassenden Demokratisierungsreform sollte der UNO-Sicherheitsrat im Gegenzug zu dem von der UNO-Generalversammlung akzeptiertem »Responsibility to protect«-Prinzip dieser umfassendere Partizipationsmöglichkeiten unterhalb der Schwelle einer Charta-Änderung auf der Ebene gewohnheitsrechtlicher Mechanismen ermöglichen. Denkbar wäre, der UNO-Generalversammlung umfassende politische Kontrollmöglichkeiten hinsichtlich der militärischen Operationen zuzugestehen und ihr die Möglichkeit einzuräumen, den UNO-Sicherheitsrat verbindlich zur Beendigung oder auch zur Aufnahme eines Einsatzes gemäß des »Uniting for Peace«-Mechanismus ${ }^{25}$ aufzufordern. Diese Partizipationsformen könnten zugleich als Grundlage für später noch weiterführende und in der UNO-Charta zu kodifizierende demokratische Willensbildungsstrukturen in dieser Weltorganisation dienen. Denn gewohnheitsrechtlich herausgebildete Normen sind aufgrund der ihnen immanenten Akzeptanz durch die beteiligten Akteure eher kodifizierbar als im Staatenverkehr noch nicht praktizierte Verfahren.

Auch müssen bis zur Umsetzung einer umfassenden Demokratisierungsreform - unter Umständen auch darüber hinaus - deutsche Beteiligungen an UN-geführten Einsätzen jeweils

25 Im Kontext des Korea-Krieges verabschiedete die UNO-Generalversammlung anstelle des blockierten UNO-Sicherheitsrats die Resolution 377 (1950):

»Falls der Sicherheitsrat aufgrund mangelnder Einstimmigkeit seiner ständigen Mitglieder es in einem Fall offenbarer Bedrohung des Friedens, eines Friedensbruchs oder einer Angriffshandlung unterlässt, seine primäre Verantwortung für die Aufrechterhaltung des internationalen Friedens und der Sicherheit nachzukommen, soll die Generalversammlung unverzüglich die Angelegenheit mit der Aussicht beraten, den Mitgliedern geeignete Empfehlungen für Kollektivmaßnahmen zu geben, einschließlich im Falle des Friedensbruches oder einer Angriffshandlung für den Gebrauch bewaffneter Kräfte, wenn notwendig, um den internationalen Frieden und die internationale Sicherheit aufrechtzuerhalten oder wiederherzustellen." einer genauen Einzelfallprüfung unterzogen werden. Hierzu böte es sich an, einen Kriterienkatalog zu entwickeln, anhand dessen man konsequent, transparent und berechenbar über jeden Einzelfall entscheiden könnte.

Als Leitfragen für den Kriterienkatalog könnten folgende Überlegungen dienen:

- Sind zuvor alle nichtmilitärischen - Instrumente ernsthaft und erschöpfend angewendet worden (Ultima Ratio)?

- Unter welchen Umständen kommen entsprechende UNOResolutionen zu einer UN-geführten Mission zustande (rechte Absicht)?

- Wie lautet der genaue Einsatzauftrag der entsprechenden UNO-Resolution - Friedenswahrung oder Friedenserzwingung (Interventionsqualität)?

- Welche Staaten stellen der UNO welche militärischen (standby) Kapazitäten zur Verfügung (Engagement)?

- Wie hoch sind die Erfolgsaussichten eines UN-Einsatzes einzuschätzen (begründete Erfolgsaussichten)?

- Sind zivile Opfer durch den Einsatz zu befürchten (Menschenrechte und Humanitäres Völkerrecht)?

- Ist der militärische Einsatz an einen zivilen Wiederaufbauplan für die Zeit nach dem militärischen Einsatz gekoppelt - am besten in der selben UN-Resolution? Sind dafür die Finanzen und das Personal bereits festgelegt (ziviler Aufbau und Rückfallprävention)?

Bei Intervention in einen innerstaatlichen Konflikt zur Beendigung von Völkermord ist die Messlatte noch höher zu legen:

- Wird das Recht auf politische, gesellschaftliche und wirtschaftliche Selbstbestimmung sowie die territoriale Integrität des Staates, in der ein Einsatz stattfinden soll, garantiert, d.h. keine Gesellschafts- und Wirtschaftsordnung oder Gebietsabtretung oktroyiert, gemäß dem Völkerrecht und den KSZE-Vereinbarungen (Souveränitätsprinzip)?

- Werden durch einen UN-Einsatz sezessionistische Kräfte im Einsatzgebiet direkt oder indirekt gefördert, so dass die UNO zum Exekutivorgan sezessionistischer Kräfte missbraucht werden könnte (Instrumentalisierungsgefahr)?

Wer es ernst mit der ungeteilten Wahrung der Menschenrechte - auch unter Zuhilfenahme militärischer Maßnahmen (»humanitäre Intervention «) -, meint, der muss auf einer zentralen und singulären Verantwortungs- und Durchführungsinstanz zur Wahrung der globalen kollektiven Sicherheit bestehen. Dazu gehört, zuvörderst die Willkür- und Instrumentalisierungsgefahr von UN-mandatierten Missionen oder gar unilateralen militärischen Maßnahmen anzuerkennen.

Eine Diversifikation von Verantwortungsträgern für globale Sicherheit schafft weder mehr Sicherheit für die Staaten noch für die Menschen - im Gegenteil: Man stelle sich einen Staat vor, in dem neben einer ohnmächtigen Polizei, weil einige mächtige Clans das so wollen, ein paar private Sicherheitsfirmen, geführt von eben jenen Clans, eigenmächtig für die Sicherheit der Bürger Sorge tragen wollen. Es käme zu Willkür und Unsicherheiten aufgrund unterschiedlicher Interessen und letztlich zu ausbrechenden Rivalitäten um Einflusszonen zwischen den Clans. Für diese Art von Staaten gibt es einen Begriff: Failing und failed states. 